\title{
Correlations among leaf traits provide a significant constraint on the estimate of global gross primary production
}

\author{
Y. P. Wang, ${ }^{1}$ X. J. Lu, ${ }^{2}$ I. J. Wright, ${ }^{3}$ Y. J. Dai, ${ }^{2}$ P. J. Rayner, ${ }^{4}$ and P. B. Reich ${ }^{5,6}$ \\ Received 3 August 2012; revised 6 September 2012; accepted 10 September 2012; published 10 October 2012.
}

[1] Current estimates of gross primary productivity (GPP) of the terrestrial biosphere vary widely, from 100 to $175 \mathrm{Gt}$ $\mathrm{C}$ year ${ }^{-1}$. Ecosystem GPP cannot be measured directly, and is commonly estimated using models. Among the many parameters in those models, three leaf parameters have strong influences on the modelled GPP: leaf mass per area, leaf lifespan and leaf nitrogen concentration. The first two parameters affect the modelled canopy leaf area and the last two determine the maximal leaf photosynthetic rate. Ecological studies have firmly established that these three parameters are significantly correlated at regional to global scales, but this knowledge is yet to be used in predicting global GPP. We hypothesize that incorporating multi-trait covariance can reduce uncertainties of model predictions in a way likely to provide improved realism. Using the Australian community land surface model (CABLE), we find that correlations among these three parameters reduce the variance among GPP estimates by CABLE by over $20 \%$ for shrub, $\mathrm{C} 4$ grassland and tundra, and by between $5 \%$ and $20 \%$ for most other PFTs, as compared with the simulated GPP without considering the correlations. Globally the correlations do not alter the mean but reduce the variance of modeled GPP by CABLE by $28 \%$ and result in fewer extremely high or extremely low (and unlikely) global GPP predictions. Therefore correlations among the three leaf parameters, and possibly other parameters, can be used as a significant constraint on the estimates of model parameters or predictions by those models. Citation: Wang, Y. P., X. J. Lu, I. J. Wright, Y. J. Dai, P. J. Rayner, and P. B. Reich (2012), Correlations among leaf traits provide a significant constraint on the estimate of global gross primary production, Geophys. Res. Lett., 39, L19405, doi:10.1029/2012GL053461.

\section{Introduction}

[2] Each year photosynthesis by the land biosphere fixes about one sixth of total atmospheric $\mathrm{CO}_{2}$ and a similar

\footnotetext{
${ }^{1}$ CSIRO Marine and Atmospheric Research, Aspendale, Victoria, Australia.

${ }^{2}$ College of Global Change and Earth System Science, Beijing Normal University, Beijing, China.

${ }^{3}$ Department of Biological Sciences, Macquarie University, Sydney, New South Wales, Australia.

${ }^{4}$ School of Earth Sciences, University of Melbourne, Melbourne, Victoria, Australia.

${ }^{5}$ Department of Forest Resources, University of Minnesota, Twin Cities, St. Paul, Minnesota, USA

${ }^{6}$ Hawkesbury Institute for the Environment, University of Western Sydney, Penrith, New South Wales, Australia.

Corresponding author: Y. P. Wang, CSIRO Marine and Atmospheric Research, Aspendale, Vic 3195, Australia. (yingping.wang@csiro.au)

Published in 2012 by the American Geophysical Union.
}

amount is released back to the atmosphere by respiration. Because these two fluxes occur simultaneously it has not been possible to measure photosynthesis separately from respiration in the field at ecosystem scale. The gross primary production (GPP) of the land biosphere defined as the total amount of carbon fixed by photosynthesis can be estimated using various approaches. Process-based models calculate GPP using either the biochemical model of leaf photosynthesis [see Farquhar et al., 1980] or the light use efficiency model [Turner et al., 2006]. Another approach is to use the relationships between various environmental variables and GPP derived from eddy flux measurements [e.g., Beer et al., 2010]. A third approach is to use other tracers, such as oxygen isotopes in $\mathrm{CO}_{2}$ or carbonyl sulfide, to infer GPP of the terrestrial biosphere [see Campbell et al., 2008; Welp et al., 2011]. Because of the large uncertainties in both the process-based and empirical models, GPP predictions obtained by the first approach vary widely, e.g. from 100 to $150 \mathrm{Gt} C$ year $^{-1}$ [Piao et al., 2010]. Using the second approach, Beer et al. [2010] estimated global annual GPP to average $123 \pm 8 \mathrm{Gt} \mathrm{C}$ year $^{-1}$ between 1990 to 2008, which is far lower (and indeed inconsistent) with the very recent estimates of 150 to $175 \mathrm{Gt} \mathrm{C}$ year $^{-1}$, made by Welp et al. [2011] using the third approach.

[3] Total canopy leaf area and maximum photosynthetic rate (photosynthetic "capacity") are two major drivers of GPP for terrestrial ecosystems [see Schulze et al., 1994; Reich, 2012]. Photosynthetic capacity is closely related to leaf nitrogen per unit leaf area that is the product of leaf nitrogen concentration, $n$ in $\mathrm{g}$ nitrogen/g dry matter and leaf mass per unit area $m$ in $\mathrm{g}$ dry matter $\mathrm{m}^{-2}$ ) [Evans, 1989; Reich et al., 1997]. Total canopy mass is largely a function of the fraction of plant carbon allocated to leaves, and of leaf lifespan [Reich et al., 1992]. In turn, total canopy leaf area is a function of canopy mass and leaf mass per area. Over the last decade or so, globally-consistent correlations have been demonstrated among leaf $\mathrm{N}$ concentration $(n)$, leaf lifespan $(\tau)$ and leaf mass per area $(m)$ [Reich et al., 1997; Wright et al., 2004]. They are: $\tau$ and $m$ being positively correlated, and both $\tau$ or $m$ being negatively correlated to $n$. Because these three traits are key parameters in global models for estimating GPP, we expected that their correlations would help us narrow down the range of estimates of GPP for different plant functional types (PFTs) and globally. This had not been explored before. We hypothesize that this can be useful in a modelling context because most studies on parameter estimation using global land models have assumed, a priori, that model parameters are independent, and showed that current observations can constrain only a small fraction of the large numbers of parameters in those models [Rayner et al., 2005; Wang et al., 2009; Ziehn et al., 2011]. If instead, incorporating multi-trait covariance can 
reduce uncertainties of model parameter estimates, predictions made by models may have improved realism, as captured by reduced variance in those predictions.

[4] The objectives of this study are (1) to quantify the degree to which correlations among the three leaf traits constrain GPP estimates for different PFTs, individually and in total; (2) to quantify the probability of global GPP being 150 to $175 \mathrm{Gt} \mathrm{C}^{\mathrm{C}}$ year $^{-1}$ at present. We used a process-based global land surface model, the Australian community land surface model (CABLE) to estimate GPP. While keeping all other model parameters unchanged, we use CABLE to compute GPP for given values of three leaf traits $(\tau, m, n)$ that are generated using a random number generator with or without taking account of the correlations. By comparing the differences in variance of the estimated GPP for the uncorrelated or correlated values of three leaf traits, we can estimate how much the correlations among the three leaf traits can reduce the uncertainties of the estimated GPP for different PFTs, or globally.

\section{Method}

[5] The CABLE model (see Kowalczyk et al. [2006] and Wang et al. [2010, 2011] for further details) is used to simulate GPP. In CABLE the vegetation is divided into 10 different PFTs based on the $1^{\circ}$ by $1^{\circ}$ International Geosphere and Biosphere Program vegetation classification [Loveland et al., 2000]. The meteorological forcing from the global soil wetness project II (GSWPII) [see Dirmeyer et al., 2006] for 1990 was used as input to CABLE. Values for vegetation and soil model parameters used in this study are listed in Kowalczyk et al. [2006] except $m, \tau$ and $n$.

[6] Using the means, variance of means and co-variances of the three leaf parameters as estimated for each of 10 PFTs based on the GLOPNET dataset [Wright et al., 2004] (see Table S1 in Text S1 of the auxiliary material), we used a random number generator to generate 500 different combination of $m, \tau$ and $n$ by either setting all covariances to zero ("uncorrelated case") or using the covariances from the observed data (see Table S1 in Text S1), the "correlated case". "Here variance of a model parameter is estimated as the standard deviation of the mean, or standard error of observations for the corresponding leaf trait.

[7] To generate a random realization of each parameter set, we constructed a covariance matrix $\mathbf{C}$ using the prescribed correlations and variances (see Table S1 in Text S1). A matrix is calculated as such that $\mathbf{C}=\mathbf{M}^{\mathrm{T}} \mathbf{M}$. $\mathbf{M}$ is often called matrix square root of $\mathbf{C}$, and may not be unique. Here we calculated it as $\mathbf{M}=\sqrt{\Lambda} \mathbf{Y}$ where $\sqrt{\Lambda}$ is a diagonal matrix of the eigen-values of $\mathbf{C}$ and $\mathbf{Y}$ the corresponding eigen-vectors. We generate a realization $\vec{v}_{i}=\vec{v}_{0}+M \vec{z}$ where $\vec{v}_{0}$ the vector of estimated trait values and $\vec{z}$ a vector of independent univariate normal random numbers. It can be shown that the population mean and covariance of these realization are $\vec{v}_{0}$ and $\mathbf{C}$ respectively.

[8] For each of 500 combinations, we ran CABLE by recycling the meteorological forcing from GSWPII for 1990 until annual GPP at every land cell reached its steady state value, which is defined as the difference in GPP between two successive runs is less than $0.01 \%$ for any land point. All

\footnotetext{
${ }^{1}$ Auxiliary materials are available in the HTML. doi:10.1029/ 2012GL053461.
}

estimates of GPP presented in this paper are steady state annual values.

\section{Dependence of GPP on the Leaf Traits}

[9] Gross primary production, GPP depends on canopy leaf area, and maximum carboxylation rate $\left(v_{\mathrm{cmax}}\right)$ and maximum rate of potential electron transport $\left(j_{\max }\right)$ at a leaf temperature of $25^{\circ} \mathrm{C}$. Canopy leaf area in CABLE is modelled as

$$
\frac{d(L m)}{d t}=f_{l} N P P / c-L m / \tau f_{T}
$$

where $L$ is canopy leaf area index, $m$ is the leaf mass per area ( $\mathrm{g}$ dry matter $\left.\mathrm{m}^{-2}\right)$, NPP is net primary production $\left(\mathrm{g} \mathrm{C} \mathrm{m}^{-2} \mathrm{~d}^{-1}\right)$, $c$ is the carbon fraction of dry biomass ( $=0.5$ in this study), $f_{1}$ is the fraction of NPP allocated to leaf and depends on leaf phenology [see Wang et al., 2010], $f_{\mathrm{T}}$ is the function describing the dependence of leaf lifespan on leaf temperature [see Wang et al., 2010]. Here we estimated the mean leaf turnover rate constant as the inverse of leaf life span, or $1 / \tau$. NPP is estimated as

$$
N P P=G P P\left(L, v_{c \max }, j_{\max }\right)-R_{m}(L, m n)-R_{g}
$$

where $R_{\mathrm{m}}$ and $R_{\mathrm{g}}$ are the maintenance and growth respiration of leaf, wood and roots.

[10] Parameters $v_{\text {cmax }}$ and $j_{\max }$ are estimated as a function of leaf nitrogen per unit leaf area. That is

$$
\begin{gathered}
v_{c \text { max }}=a+b m n \\
j_{\text {max }}=2 v_{c \text { max }}
\end{gathered}
$$

where $a$ and $b$ are two empirical coefficients with the values from Kattge et al. [2009] for different PFTs except C4 plants and tundra. We used the values of $a$ and $b$ of $\mathrm{C} 3$ grass as no estimates were provided by Kattge et al. [2009] for C4 plants, and values of Shrub for Tundra (see Table S2).

[11] In CABLE, GPP and $R_{\mathrm{m}}$ are calculated hourly, $R_{\mathrm{g}}$ is calculated daily. Equation (1) is integrated forward at daily time step.

[12] Total variance of the simulated GPP is calculated for each land cell, each PFT or globally. The total variance of GPP can also be decomposed into the contribution of variance from each leaf trait and the covariance among three leaf traits (see Text S1 in Text S1). For the uncorrelated case, the covariance always is zero.

\section{Results}

[13] We evaluated the variance and co-variance terms for each PFT by computing the first derivatives using the finite difference method. The covariance is negative for all PFTs (see Figure 1), suggesting that taking the correlation structure into account will reduce the uncertainty of the estimated GPP for all PFTs. The absolute reduction in total variance due to the correlations is relatively larger for deciduous needleleaf forest, C3 and C4 grasslands than other PFTs. However the results as shown in Figure 1 are quantitatively accurate only around mean GPP for each PFT, and can be biased for other values of GPP if the first derivatives in equation (S3) vary with the independent variables. In the following, we will present the mean and variance of the 


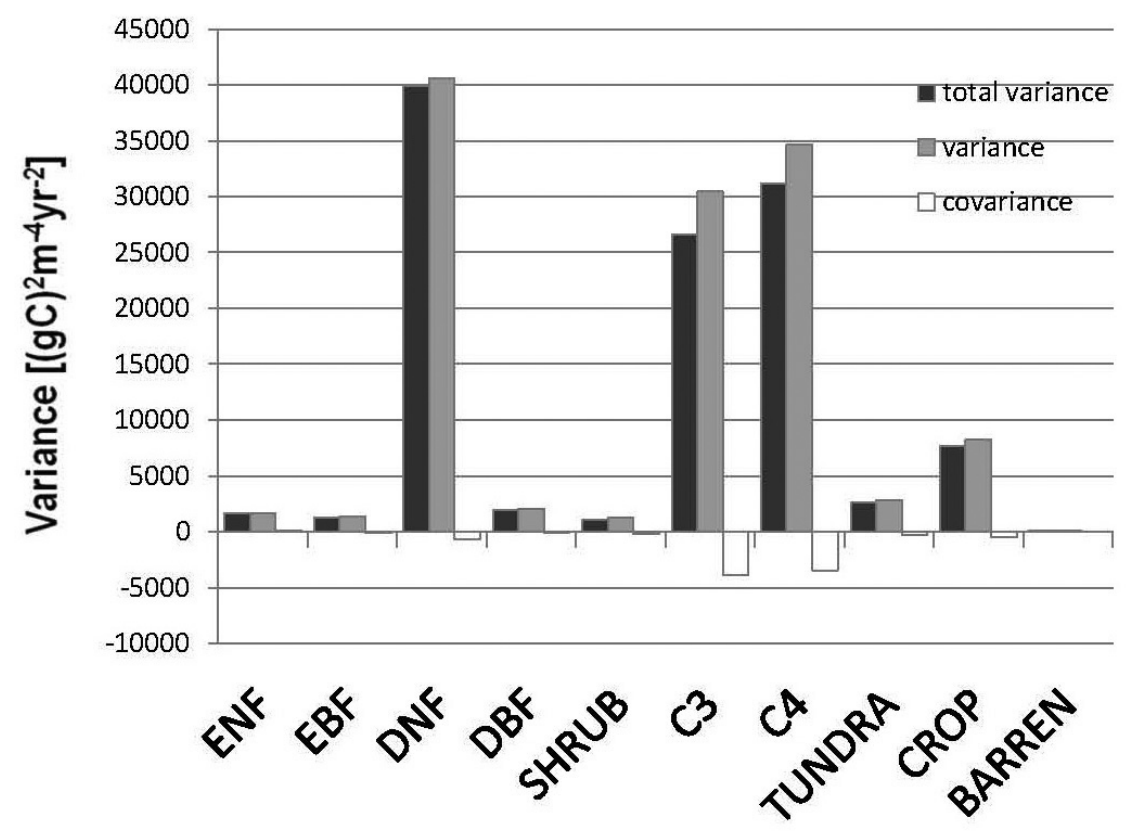

Figure 1. Total variance, variance and covariance of the estimated mean GPP for different plant functional types as calculated using equation (S3). See Table S1 in Text S1 for the definitions of all acronyms in the x-axis label.

simulated GPP by CABLE from 500 sets of leaf trait values, for both the correlated or uncorrelated cases.

[14] The global pattern of the mean GPP as calculated by CABLE for the uncorrelated case (see Figure 2a) is quite similar to Beer et al. [2010] using empirical models (see Figure 2b) except evergreen needle leaf forest, C3 crops and barren land. The mean GPP as estimated by CABLE for the uncorrelated case is quite similar to those for the correlated case for all 10 PFTs. The tropical evergreen forest has the highest mean annual GPP, about $3200 \mathrm{~g} \mathrm{C} \mathrm{m}^{-2}$ year $^{-1}$, similar to the estimate by Kattge et al. [2009] or Beer et al. [2010] (see Figure 2b).

[15] As compared with the estimates of Beer et al. [2010] for evergreen needle leaf forest and $\mathrm{C} 3 \mathrm{crop}$, the differences in the mean GPP largely result from the differences in the mean leaf lifespan. The arithmetic mean leaf life span as estimated by Wright et al. [2004] is 3.78 years for evergreen needle leaf forest and 0.29 years for C3 crop (see Table S1 in Text S1), as compared with 2 years and 0.46 years as the default values in CABLE that was calibrated using the GPP estimates of Beer et al. [2010]. The relative differences between the default values in CABLE and the means of those three leaf traits from Wright et al. [2004] often are largest for $\tau$. In general, the default values of $\tau$ in CABLE are smaller for forest types, and larger for other vegetation types than those from Wright et al. [2004].

[16] However the uncertainties of the estimated GPP are quite large, particularly for the regions in Brazil, Central Africa and south China, and south-east USA (see Figure 3a), where the mean GPPs also are quite high $\left(>1200 \mathrm{~g} \mathrm{C} \mathrm{m}^{-2}\right.$ year $\left.^{-1}\right)$ (see Figure 2a). Therefore the uncertainties in the estimated GPPs in those regions contribute to a significant fraction $(>40 \%)$ of the total variance of global GPP for the uncorrelated case. If the correlations among the three leaf traits are considered, the variance of simulated GPP by CABLE is reduced by more than $50 \%$ for some shrub lands in Northern Australia and South Africa, or by more than $20 \%$ for most tropical C4 grassland in South America, Africa and Australia, as well as tundra at northern high latitudes (see Figure $3 \mathrm{~b}$ ). The reduction in variance by taking into account the correlations among the three leaf traits is significant at $99 \%$ level for much of the global land surface (see Figure 3c).

[17] Figure 4 (top) shows that the frequency distribution of global GPP estimates from the uncorrelated case is higher than that from the correlated case at GPP $>160 \mathrm{Gt} \mathrm{C}$ year $^{-1}$ or $<130 \mathrm{Gt} \mathrm{C}$ year $^{-1}$, and is lower than that from the correlated case at GPP between 130-145 Gt C year ${ }^{-1}$. Overall the correlations among the three leaf traits reduce the variance of estimated global GPP by CABLE by $28 \%$. The global GPP for the uncorrelated case is $137.3 \pm 9.2 \mathrm{Gt} \mathrm{C}$ year $^{-1}$, as compared to $137.5 \pm 7.8 \mathrm{Gt} \mathrm{C}$ year $^{-1}$ for the correlated case as simulated by CABLE.

[18] For different PFTs, the correlations among the three leaf traits reduces the total variance of the estimated GPP by CABLE by over $20 \%$ for shrub, C4 grassland and tundra, and by $5 \%$ to $20 \%$ for evergreen broadleaf forest, deciduous broadleaf forest, C3 grass, cropland and barren land. The variance reduction from the correlations is quite large for $\mathrm{C} 4$ grasslands that only have very few observations of three leaf traits. As a result, the variance of the estimated GPP is still quite large for the correlated case $\left(211 \mathrm{~g} \mathrm{C} \mathrm{m}^{-2}\right.$ year $\left.^{-1}\right)$.

[19] Our results (Figure 2b and Figure 4, bottom) show that the correlations among the leaf traits reduce the total variance of the estimated GPP but only have small influences on the mean of estimated GPP by CABLE for all different PFTs and global land biosphere. However the reduction in the total 

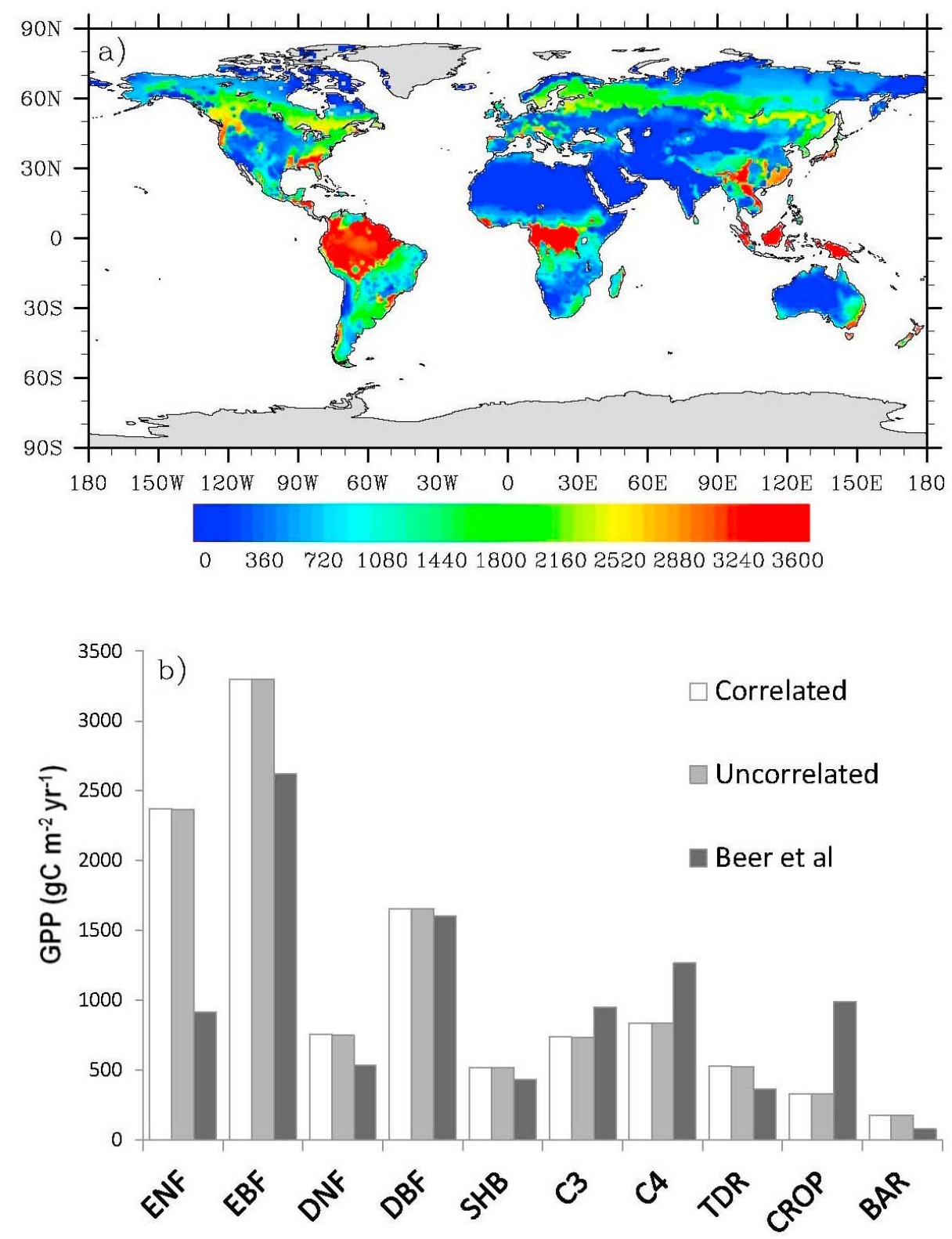

Figure 2. (a) The mean estimate of the simulated GPP by CABLE for the uncorrelated case, and (b) mean GPP of different plant functional types for the correlated and uncorrelated cases in comparison with the results from Beer et al. [2010].

variance of GPP for each PFT as shown in Figure 4 (bottom) is much larger than that in Figure 1b, because the relationship between GPP and each of the three leaf traits is nonlinear.

\section{Implications}

[20] Using the observed mean, variance and the correlations among the three key leaf traits for canopy photosynthesis, we simulated that global GPP of land biosphere is $137.5 \pm 7.8 \mathrm{Gt} \mathrm{C}_{\mathrm{Cear}}{ }^{-1}$, which is higher than the estimated GPP by CABLE using default values for the three leaf traits. However the estimated mean GPP in this study is not statistically significantly different from the estimate of Beer et al. [2010] using empirical models, but is significantly lower than the best estimate of 150 to $175 \mathrm{Gt} \mathrm{C}^{\mathrm{year}}{ }^{-1}$ by Welp et al.
[2011]. Based on our results, the probability is less than $9 \%$ for GPP being from 150 to $175 \mathrm{Gt} \mathrm{C}^{\text {year }}{ }^{-1}$, and would be even lower if all other parameters in CABLE were recalibrated against the GPP estimates of Beer et al. [2010]. A full analysis of the uncertainty of GPP for different PFTs or global land biosphere will need to bring in additional constraints, such as latent heat flux and leaf area index. This will be explored in the future.

[21] Results from this study have several implications. The first one is that our results provide further support for the need of developing a trait-based classification of PFTs for global land surface models, as advocated by Reich et al. [2007]. Global land surface models use plant functional types to represent different land surface types, and each plant functional type is given a set of values for different model 

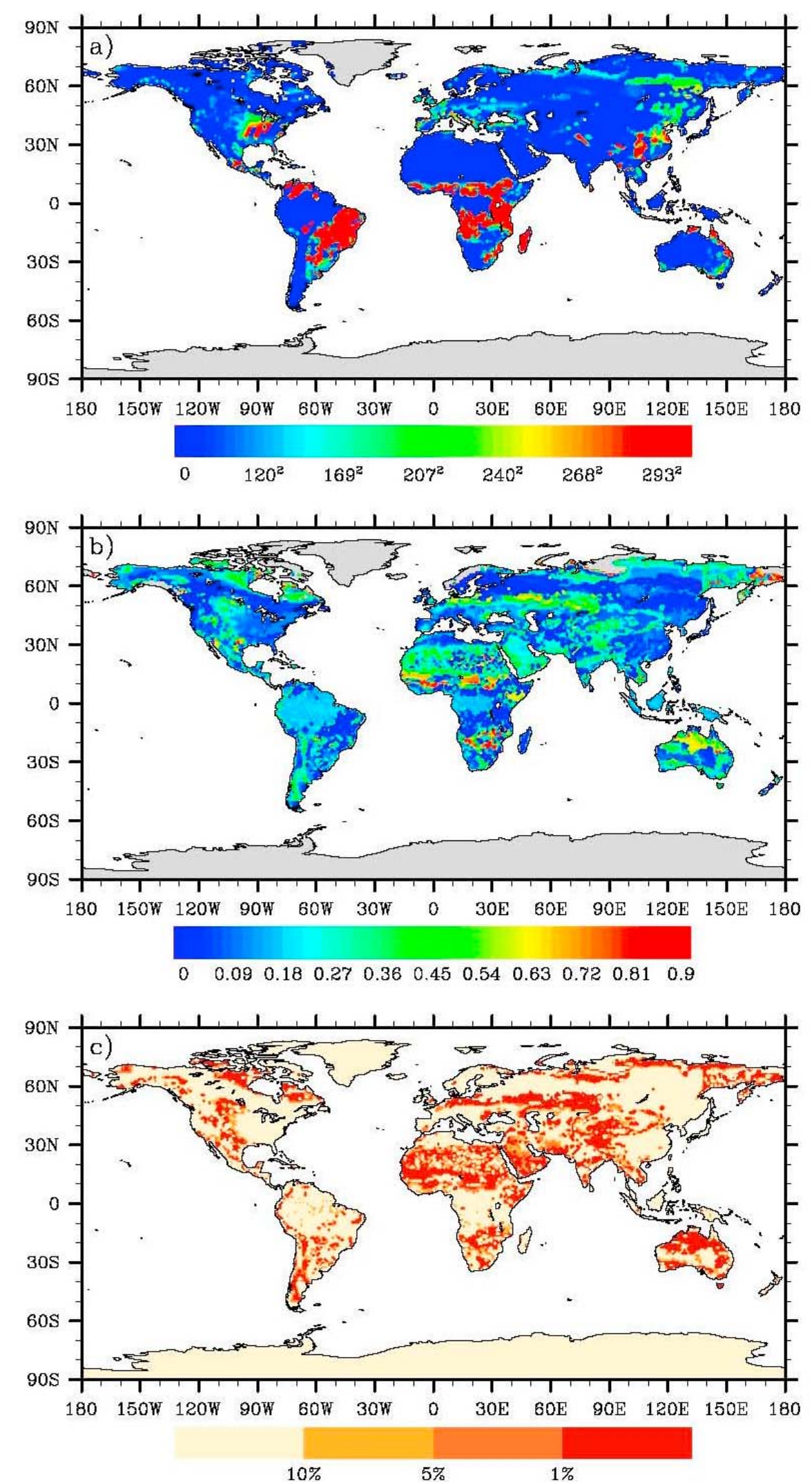

Figure 3. (a) Variance of the estimated mean GPP for the uncorrelated case in $\left(\mathrm{g} \mathrm{C}^{2} \mathrm{~m}^{-4}\right.$ year ${ }^{-2}$, (b) fractional reduction in the variance when the correlations among the three leaf traits are considered and (c) the region with variance reduction being significant at $10 \%, 5 \%$ or $1 \%$ levels (F test). 

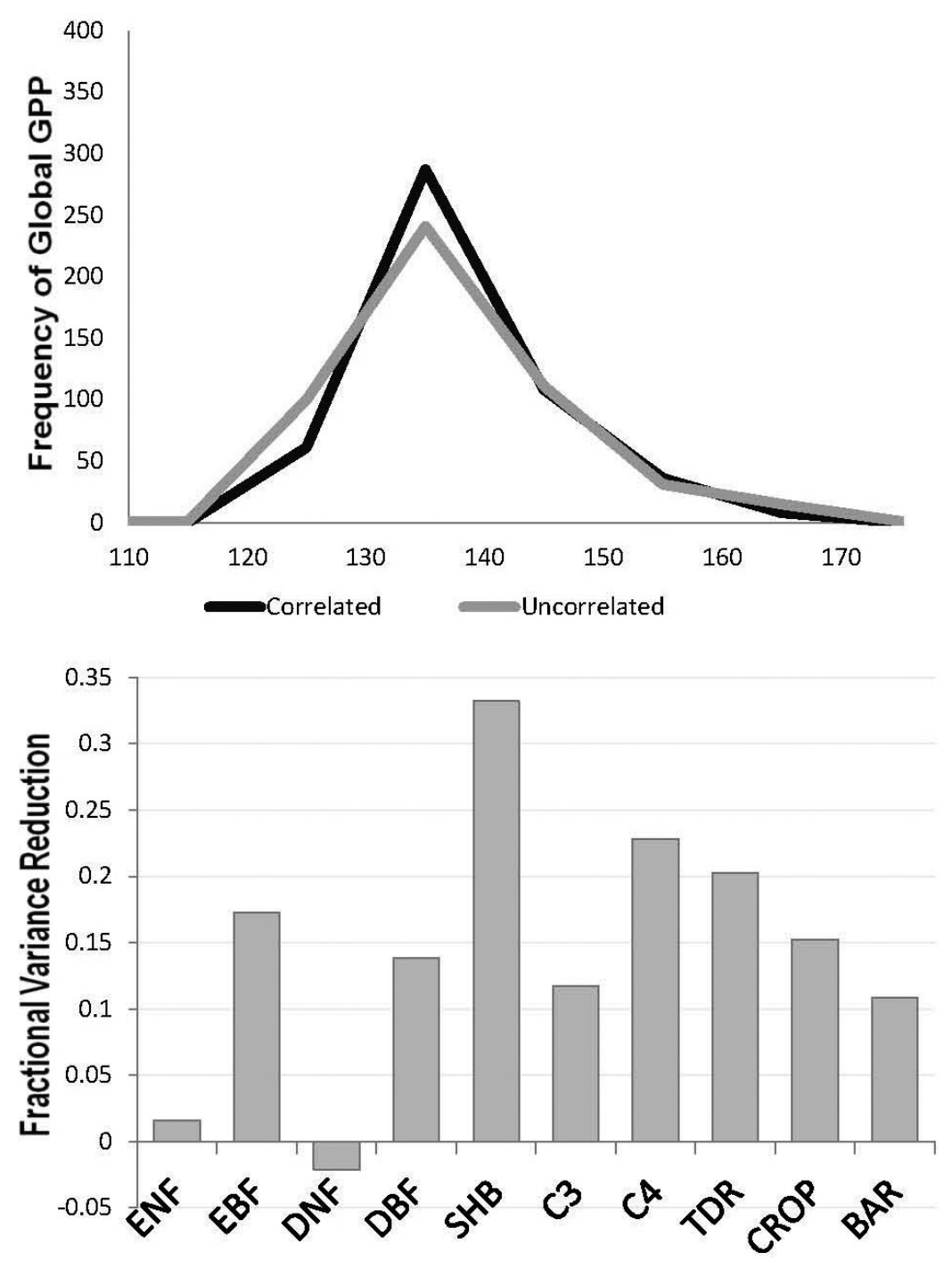

Figure 4. (top) Frequency distribution of the simulated global GPP by CABLE for the correlated and uncorrelated case (in $\mathrm{Gt} \mathrm{C}$ year ${ }^{-1}$ ), and (bottom) the fractional reduction in the variance of the estimated GPP for different plant functional types.

parameters, including the three parameters we studied here. Because of the large number of model parameters and their uncertainties, those models have high degrees of freedom, which makes model calibration quite difficult and predictions by those models are highly uncertain. By taking into account the known correlations among different model parameters we can thus reduce the degrees of freedom in the model parameter space as well as the uncertainty associated with model predictions, therefore PFT-specific effects on model output can then be better distinguished from another in global land surface modeling. The second type of implication is that use of covariance among key leaf traits can reduce uncertainties of model parameter estimates and predictions by those models. Most studies on parameter estimation using global land models have assumed, a priori, that model parameters are independent, and found that current observations can be used to constrain only a small fraction of the large numbers of parameters in those models [Rayner et al., 2005; Wang et al., 2009; Ziehn et al., 2011]. On the other hand, field observations of key leaf traits are quite comprehensive as in the GLOPNET and other datasets [see Wright et al., 2004; Kattge et al., 2011.]. There is an increasing trend towards firmly establishing at regional to global scales the general relationships among key functional traits of plants [e.g., Reich et al., 1997; Wright et al., 2004; Cornwell et al., 2008, 2009; Chave et al., 2009]. Results from this study suggest that correlations among the three leaf traits, and possibly other traits [see Ziehn et al., 2011] can be used as a significant constraint on the estimates of model parameters or predictions by those models to regional to global scales.

[22] Acknowledgments. We benefitted from discussion with Gordon Bonan and Rosie Fisher during the early stages of this study and Jens Kattge in the latter stages. We are grateful for the financial support to Xingjie Lu from the China Scholarship Council; to Ying-Ping Wang from the Department of Climate Change and Energy Efficiency, Australia; to the Australian 
Research Council for Future Fellowship funding to Ian Wright (project FT100100910) and to the Australian Research Council Professorial Fellowship to Peter Rayner (project DP1096309), and to the U.S. NSF (DEB 0620652) and Wilderness Research Foundation to Peter Reich.

[23] The Editor thanks the anonymous reviewer for assistance in evaluating this paper.

\section{References}

Beer, C., et al. (2010), Terrestrial gross carbon dioxide uptake: Global distribution and covariation with climate, Science, 329, 834-838, doi:10.1126/ science.1184984.

Campbell, J. E., et al. (2008), Photosynthetic control of atmospheric carbonyl sulfide during the growing season, Science, 322, 1085-1088, doi:10.1126/science.1164015.

Chave, J., et al. (2009), Towards a worldwide wood economics spectrum, Ecol. Lett., 12, 351-366, doi:10.1111/j.1461-0248.2009.01285.x.

Cornwell, W. K., et al. (2008), Plant species traits are the predominant control on litter decomposition rates within biomes worldwide, Ecol. Lett., 11, 1065-1071, doi:10.1111/j.1461-0248.2008.01219.x.

Cornwell, W. K., et al. (2009), Plant traits and wood fates across the global: Rotted, burned, or consumed?, Global Change Biol., 15, 2431-2449, doi:10.1111/j.1365-2486.2009.01916.x.

Dirmeyer, P. A., X. Gao, M. Zhao, Z. H. Guo, T. Oki, and N. Hanasaki (2006), GSWP-2- multimodel analysis and implications for our perception of the land surface, Bull. Am. Meteorol. Soc., 87, 1381-1397, doi:10.1175/BAMS-87-10-1381.

Evans, J. R. (1989), Photosynthesis and nitrogen relationship in leaves of $\mathrm{C}_{3}$ plants, Oecologia, 78, 9-19, doi:10.1007/BF00377192.

Farquhar, G. D., S. von Cammerer, and J. A. Berry (1980), A biochemical model of photosynthetic $\mathrm{CO}_{2}$ assimilation in leaves of $\mathrm{C}_{3}$ species, Planta, 149, 78-90, doi:10.1007/BF00386231.

Kattge, J., W. Knorr, T. Raddatz, and C. Wirth (2009), Quantifying photosynthetic capacity and its relationship to leaf nitrogen content for globalscale terrestrial biosphere models, Global Change Biol., 15, 976-991, doi:10.1111/j.1365-2486.2008.01744.x.

Kattge, J., et al. (2011), TRY - A global data base of plant traits, Global Change Biol., 17, 31 pp., doi:10.1111/j.1365-2486.2011.02451.x.

Kowalczyk, E. A., Y. P. Wang, and R. M. Law (2006), The CSIRO Atmospheric Biosphere Land Exchange (CABLE) model for use in climate models and as an offline model, Pap. 013, Commonw. Sci. and Ind. Res. Organ. Mar. and Atmos. Res., Aspendale, Vic., Australia. [Available at http://www.cmar.csiro.au/e-print/open/kowalczykea_2006a.pdf.]

Loveland, T. R., B. C. Reed, J. F. Brown, D. O. Ohlen, Z. Zhu, L. Yang, and J. W. Merchant, (2000), Development of a global land cover characteristics database and IGBP DISCover from $1 \mathrm{~km}$ AVHRR data, Int. J. Remote Sens., 6-7, 1303-1330.

Piao, S. L., et al. (2010), Forest annual carbon cost: A global-scale analysis of autotrophic respiration, Ecology, 91, 652-661, doi:10.1890/08-2176.1.

Rayner, P. J., M. Scholze, W. Knorr, T. Kaminski, R. Giering, and H. Widmann (2005), Two decades of terrestrial carbon fluxes from a carbon cycle data assimilation system, Global Biogeochem. Cycles, 19, GB2026, doi:10.1029/2004GB002254.

Reich, P. B. (2012), Key plant traits drive forest productivity, Proc. R. Soc. $B, 279,2128-2134$.

Reich, P. B., M. B. Walters, and D. S. Ellsworth (1992), Leaf life-span in relation to leaf, plant, and stand characteristics among diverse ecosystems, Ecol. Monogr., 62, 365-392, doi:10.2307/2937116.

Reich, P. B., M. B. Walters, and D. S. Ellsworth (1997), From tropics to tundra: Global convergence in plant functioning, Proc. Natl. Acad. Sci. U. S. A., 94, 13,730-13,734, doi:10.1073/pnas.94.25.13730.

Reich, P. B., I. J. Wright, and C. H. Lusk (2007), Predicting leaf physiology from simple plant and climate attributes: A global GLOPNET analysis, Ecol. Appl., 17(7), 1982-1988, doi:10.1890/06-1803.1.

Schulze, E.-D., F. M. Kelliher, C. Korner, J. Lloyd, and R. Leuning (1994), Relationships among maximum stomatal conductance, ecosystem surface conductance, carbon assimilation rate, and plant nitrogen nutrition: a global ecology scaling exercise, Annu. Rev. Ecol. Syst., 25, 629-662, doi:10.1146/annurev.es.25.110194.003213

Turner, D. P. et al. (2006), Evaluation of MODIS NPP and GPP products across multiple biomes, Remote Sensing of Environment, 102, 282-292. Wang, Y. P., C. T. Trudinger, and I. G. Enting (2009), A review on applications of model-data fusion to studies of terrestrial carbon fluxes at different scales, Agric. For. Meteorol., 149, 1829-1842, doi:10.1016/ j.agrformet.2009.07.009.

Wang, Y. P., R. M. Law, and B. Pak (2010), A global model of carbon, nitrogen and phosphorus cycles for the terrestrial biosphere, Biogeosciences, 7, 2261-2282, doi:10.5194/bg-7-2261-2010.

Wang, Y. P., E. Kowalczyk, R. Leuning, G. Abramowitz, M. R. Raupach, B. Pak, E. van Gorsel, and A. Luhar (2011), Diagnosing errors in a land surface model (CABLE) in the time and frequency domains, J. Geophys. Res., 116, G01034, doi:10.1029/2010JG001385.

Welp, L. R., et al. (2011), Interannual variability in the oxygen isotopes of atmospheric $\mathrm{CO}_{2}$ driven by El Niňo, Nature, 477, 579-582, doi:10.1038/ nature10421.

Wright, I. J., et al. (2004), The worldwide leaf economics spectrum, Nature, 428, 821-827, doi:10.1038/nature02403.

Ziehn, T., J. Kattge, W. Knorr, and M. Scholze (2011), Improving the predictability of global $\mathrm{CO} 2$ assimilation rates under climate change, Geophys. Res. Lett., 38, L10404, doi:10.1029/2011GL047182. 